\title{
History of Automatic Focusing Reflected by Patents
}

\author{
Octavian Baltag ${ }^{1,2}$ \\ ${ }^{1}$ Terraflux Control Ltd., Iasi, Romania \\ ${ }^{2}$ Department of Biomedical Engineering, “Grigore T. Popa” Univ. of Medicine and Pharmacy, Iasi, Romania
}

\section{Email address:}

octavian.baltag@bioinginerie.ro

\section{To cite this article:}

Octavian Baltag. History of Automatic Focusing Reflected by Patents. Science Innovation. Vol. 3, No. 1, 2015, pp. 1-17.

doi: 10.11648/j.si.20150301.11

\begin{abstract}
The work presents the evolution of a technical idea concerning the automatic image focusing in photo cameras, TV cameras and camcorder-type recording cameras. The work aims to present both the evolution of the technical solutions, and a Romanian priority concerning the automatic focusing reached by the technologies of the optical and electronic industries of image taking only 15 years after having been patented in Romania the most utilized principles, namely the dynamic analysis of image contrast, a principle used in commercialized cameras after the years ' 80 .
\end{abstract}

Keywords: Autofocus, Patents, History, Contrast Image, Phase Contrast, Photocamera

\section{Introduction}

In order to obtain sharp images in the photo or video cameras, an operation called adjustment for definition or focusing is necessary. By this operation the image sharpness is adjusted up to a level perceived as correct by the operator (photographer). The notion of adjustment for definition or focusing is used in the instrumental optics to observe the images through field glass, lunette, telescope, microscope, laparoscope, photo cameras, and image projection on a screen. As for the eye, also considered as an optical instrument, the term eye accommodation is used, equivalent to the focusing notion from instrumental optics. The sight results from the analysis of the image formed on retina, an image that can be either clear or diffuse, depending on the accommodation. The mechanism of eye is part of a chain of automatisms specific to living systems, such that we obtain on retina a clear image of the object on which our attention and sight are simultaneously concentrated. The accommodation mechanism consists in the modification of crystalline lens convergence, such that the obtained image has sharp contours, with the highest contrast possible; the other images belonging to objects located in remote or close planes will have diffuse contours. The same phenomenon is noticed in the photo cameras that do not have an optical system consisting of lenses, namely in "camera obscura" without an objective (pinhole). There are situations when the image of the object on which our attention is directed is the result of a reflection in the mirror, window or any other reflecting surface. The eye and the nervous system work together such that, irrespective of the distance, the accommodation is performed automatically, through a process whose mechanisms are very complex. This process analyzes the image projected on the retina and corrects the eye crystalline through the ciliary muscles to produce an image that is interpreted by the nervous system as having maximum sharpness. Certainly, the sight defects can also intervene, but the neuromuscular system interprets and establishes which image can be considered as "maximum-maximorum" sharp image, taking into account those defects. When introducing a correction (by means of lenses, for example), the image quality improves, the focusing- accommodation mechanism working in the same complex loop of neuromotor reaction of perception and ocular system.

A relatively simple focusing method is based on the following principle, resulted from the laws of the geometric optics: the image of a point of a scene is correctly focused only for a one objective-image plane distance; this distance is in direct relation with the focal length of the objective and the objective- scene distance. The optimum distance for a correct focusing can be determined for a certain objective aperture; accordingly, the spectrum of the formed image will contain the higher frequencies, the better the image is focused (sharper and with the highest contrast). As a rule, the optimum distance is found through repeated trials, the dichotomy being the fastest manner to find the optimum focusing. The method was presented and applied in certain patents, in some applications 
in robotics and television cameras. There are several focusing methods for the photographic images, each one using specific physical principles, mechanisms and software:

- fix focusing;

- manual focusing;

- automatic focusing that can be of two types: active and passive;

- hybrid focusing that uses the active and passive methods together.

The automatic focusing makes use of a system called "autofocus - AF" or "mis au point automatique" (French). This system analyzes the quality of the image projected on an image analyzer prior to command the exposure of the photographic film or of the image sensor. There are several systems of image analysis, determined by the utilized principle. We can thus distinguish two methods: active focusing and passive focusing.

The active focusing refers to a system through which one measure the distance (using an active source of ultrasonic or IR energy) between the object situated in the main plane, and the photo camera, and depending on this distance, the objective focusing is modified either by shifting the entire objective, or one lens or group of lenses destined for focusing. The distance is measured using several principles: US or microwave radar system, active optical telemeter with IR radiations or another radiation from the visible range; another method concerns the optical telemeter (range finder) that transmits a complex image (matrix) consisting of several luminous points projected on the aimed object.

The autofocus passive systems are named like this since they do not make use of active principles (ultra-acoustic waves, infrared radiations, etc) in order to obtain information concerning the object-camera distance. At the same time, these systems only use information that can be found in the image projected by an optical system on the sensitive element playing the role of analyzer, or even on the sensitive element used to take the image. Even if it provides remarkable performances, there are situations when the passive $\mathrm{AF}$ system needs to be assisted by secondary sources when the image is not bright; this is the case of taking pictures in the dark using the flashes for focusing, or the image projection and analysis of a matrix on the aimed subject. The main AF passive systems use two principles: phase detection and image contrast analysis.

In some special applications (robotics) the hybrid autofocus systems are utilized that use simultaneously:

- active and passive methods;

- phase contrast and image contrast analysis.

\section{Short History of the Old Technology}

The history of the development of the automatic focusing systems starts in 1932. The first mention of a system destined to automatic focusing of a photographical image is found in the US Patent from 1932 belonging to an illustrious American inventor of Armenian origin named [1]. The patent is entitled: "Self-focusing camera"; the patent application was filed on the $16^{\text {th }}$ of June 1931 and the patent was granted on the $12^{\text {th }}$ of July 1932 with the number 1,866,581 (see Figure 1).

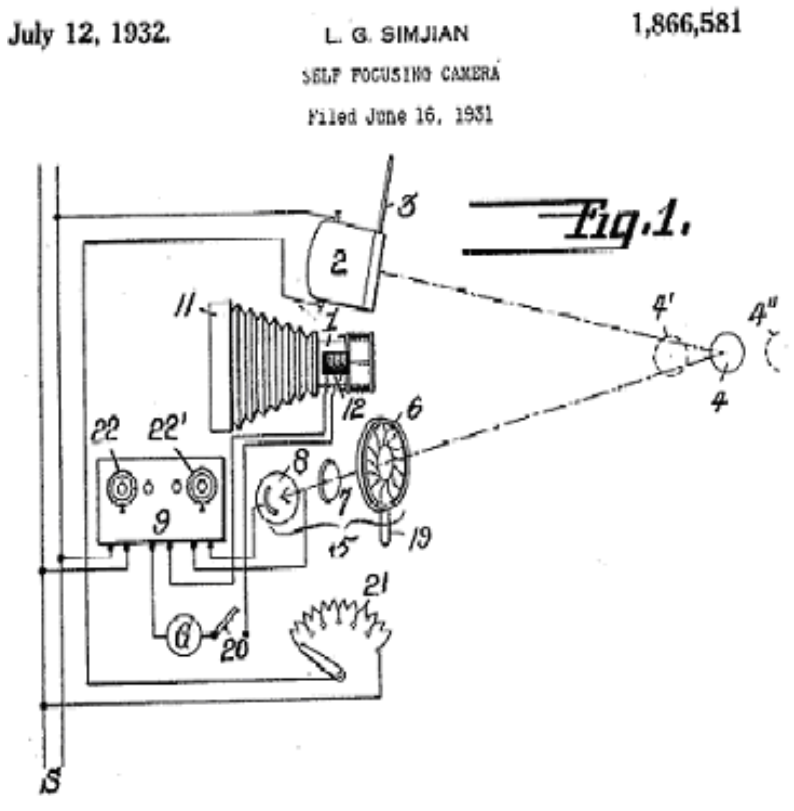

Figure 1. Self-Focusing camera

The system uses the fact that the illumination depends in inverse proportion on the squared distance between the subject and the light source; the system is endowed with a photoelectric cell through which the subject illumination is measured. The signal generated by the photocell is amplified and it commands an electromagnet that modifies the relative position of an objective lens with respect to the photographed subject and the photo plate. To make it work, it is necessary to correctly focus the photo camera at the beginning, before starting to take photos, such that the image has a maximum sharpness. For this purpose, the camera is adjusted for the initial photographing distance by manually adjusting the iris diaphragm of the shutter; in this way the illumination of the photocell and the initial position of the objective are adequately modified for a right image focusing. After these adjustments, the control system and the camera are ready to perform the automatic focusing when the subject moves closer or farther along the camera direction. The system is also equipped with a galvanometer that measures the current intensity through the electromagnet, giving thus an indication on the focusing quality.

On presents a synthesis of the main technical solutions and automatic focusing systems with applications in photography, television and cine-cameras, also specifying the year of patent granting. The synthesis refers to the period 1932-1973, a period which also includes the year 1965, when at OSIM (State Office for Inventions and Trademarks) Romania was filed the patent application for the invention "Video-captor apparatus with automatic focusing".

L., Simijian, US patent 1,866,581, (1932), Self-focusing camera [1]. The system uses the fact that illumination depends in inverse proportion on the squared distance between the subject and the light source, and a photocell that measures the 
subject illumination.

G.L., Beers US patent 2,403,628, (1946), Television pickup control system [2]. Measurement of incremental variation at different time intervals corresponding to a different optical focusing of the spectral component of the video signal frequency.

R.K., Orthuber, US patent 2,831,057, (1958), Automatic focus adjuster [3]. Three video-captor cameras with three identical optical systems are used, which provides three different focusing levels: focusing in front of the main surface, in the main surface and behind the main surface. The analysis of the level of the video-signals corresponding to the two extreme cameras determines the sense of simultaneous motion of the three objectives, the optimum focusing position of the central camera being thus obtained.

H.H., Salinger, US patent 2,838,600, (1958), Focusing adjusting system [4]. Two camera tubes are used, a primary one for image transmission and a secondary one used for focusing analysis and control. The image projected in the secondary camera tube is periodically modulated through focusing modulation by means of two lenses that periodically alternate through the optical system resulting in two images: one focused in front of the camera tube photocathode and another one behind it. The resulted video signal contains a different video frequency spectrum, depending on the focusing quality. A circuit sensitive to spectral changes of the video signal controls the direction in which the correction of the principal camera objective focusing is made.

W.L. Steiner, US patent 3,211,831, (1965), Automatic focus system and method [5]. Autocorrelation of frequency spectrum of two successive images with two different focusing, one of the images being saved in a tube with electrostatic memory. The focusing correction depends on the amplitude of the second derivative of the successive correlation coefficients. The optimum focusing corresponds to the maximum value of the correlation coefficient, while the variation of the second derivative amplitude indicates the direction in which the optical correction must be applied.

O. Baltag, RO patent 44,277, (filled in 1965, and patented in 1966) Device for automatic focusing [6]. It makes use of electronic circuits for the dynamic analysis of the image contrast from a pre-selected region and adjusts the objective position with respect to image transducer in successive steps until one obtains the maximum contrast of the electronic image corresponding to maximum contrast of the optical image.

F. Biedermaer et al., US patent 3,274,914, (1966), Camera focusing device [7]. It uses the optical telemeter whose main axis coincides with the camera main axis. Two bridge-connected photo-sensitive matrices generate an electric signal used to indicate the optimum focusing. The system is not automated in the AF known sense.

A.B. Pagel, US patent 3,442,193, (1969), Automatic focusing system triangulation [8]. It uses a light beam transmitted by camera to the photographed subject, the beam being reflected back to the camera and detected by a photosensitive element. The angle between the sent and reflected beams represents a measure of the camera-subject distance, being used as focusing error parameter, which is corrected by shifting the objective.

C.E. Thomas et al, US patent 3,522,764, (1969), Automatic focusing apparatus [9]. Destined to projectors, it compares the frequency spectrum of two successive images and applies the correction for automatic focusing.

G.L. Martin, US patent 3,708,619, (1973), Automatic focusing of optical systems [10]. It uses an optical corrector consisting of a double mirror to obtain two spatially separated images that are projected on two photosensitive elements, by whose means the size difference between the two images is detected. The signal resulted from the two photo elements represents the control signal for the focusing lens group.

There is no information concerning the utilization and application of the automatic focusing in the television transmissions, but some information from 6's years mention the existence on some TV cameras of some analogue indicator instruments that supply information about the video signal spectrum component. From these indications the camera operator can draw a conclusion concerning the focusing quality, as the correct focusing means the presence of high frequencies in the video spectrum.

The technological level on those years only permitted an analogue processing of the signal supplied by the optical sensors that integrally analyzed the image projected on sensors. The only patent referring to the contrast analysis of an image segment is dated 1965 - Romania, which used the dynamic analysis of a part of the image, projected in a shooting camera, namely part of the exploration of a TV line.

After the ' 70 , until the moment when the AF systems appeared on the market and were used, several ingenious technical solutions were imagined and recorded, destined to both TV cameras and shooting and cine-cameras. These solutions were materialized in various prototypes presented at different photographic exhibitions. The evolution of optical and electronic technologies, apparition of integrated circuits and microactuators, permitted the application and integration in photographic cameras of new technical solutions, most of them proprietary.

\section{Evolution of Autofocusing Photographic Cameras}

Starting from the end of 70's, many manufacturers experimented the automatic focusing on various prototypes of compact cameras and SLR; this brought in the next decade the domination on the market of completely automated cameras, having also integrated the "AF" function of automatic focusing. The first mention of the utilization and application of the automatic focusing in a photo camera appears in 1963, when Canon Inc. introduces at the fair Photokina '63 from Cologne, a prototype of photo camera having an automatic focusing system; the principle of operation is not known and, as far as we know, no demonstrations with this camera were made. 

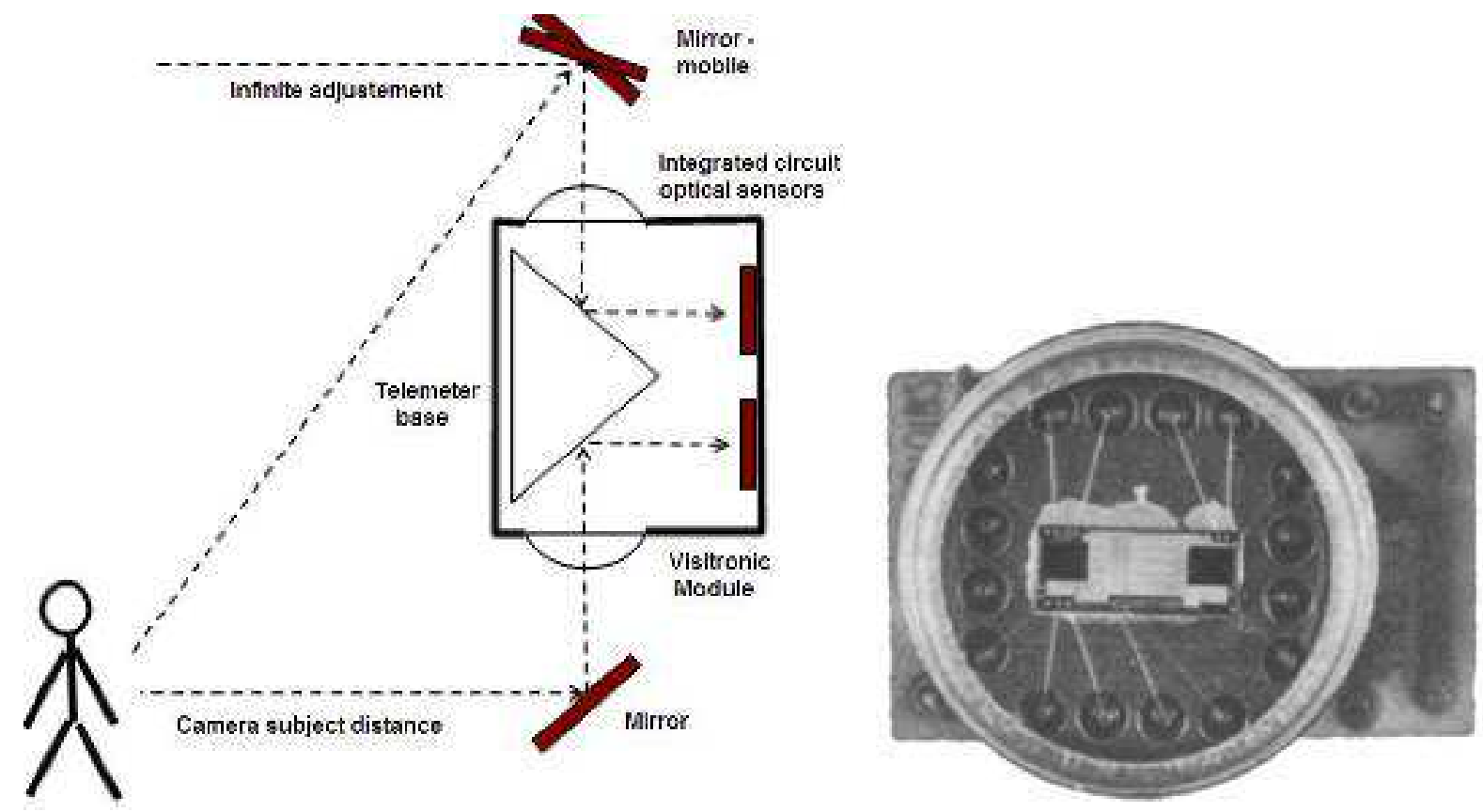

Figure 2. Principle used by Visitronic, and the optical sensor

At the end of the 1975, the Hengniweir Company accomplishes and proclaims through Honeywell, USA, the first operational AF device of automatic focusing that makes use of a double optical system, with telemetry through triangulation, known as "Visitronic System" or, in brief, VAF (Visitronic Auto Focus). It is delivered as an AF module to various manufacturers, who associate it with various components of external control and execution.

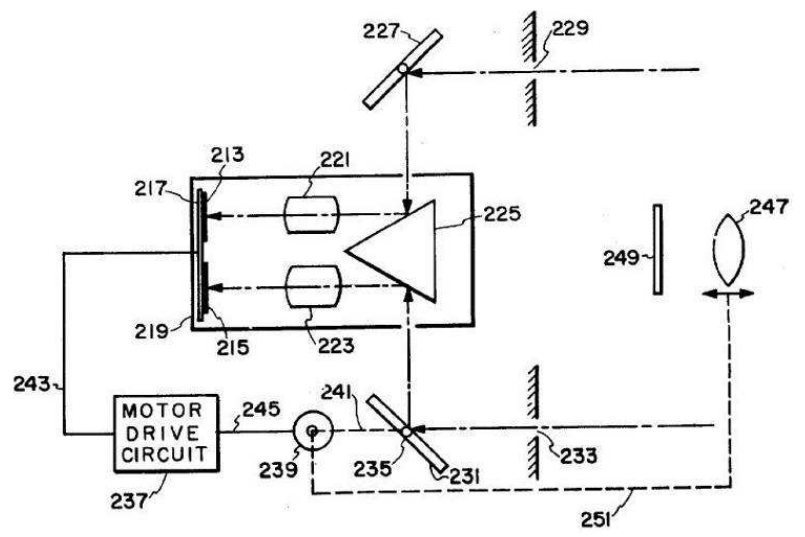

Figure 3. Visitronic Auto Focus principle

No Visitronic photo camera was produced, but the $V A F$ system remained. This is a system consisting of an integrated circuit with photo sensors, and a stereoscopic optical system with two paths for image transmission. Figure 2 presents schematically the utilized principle, while Figure 3 gives a detail from the US Patent 4,002,899 (patent filed Oct. 1975) in which the solution of this telemetric system is described in details [11].
The integrated circuit is used to determine the self-correlation of the detected optical signals. The integrated circuit consists of two separated matrices, each of them including five photosensitive elements connected to an analogue circuit for signal amplification and processing. The scene image is transmitted through two frontally open windows located at the margins of the assembly; the device must be symmetrically installed with respect to the camera objective. The image is transmitted by means of two pairs of mirrors, one fixed and tilted at $45^{\circ}$ with respect to the optic axis, the other one mobile around the angle of $45^{\circ}$.

To adjust the optical and electronic systems for a scene located at infinity, the mobile mirror is placed at $45^{\circ}$. The picked up images are transmitted by the two photosensitive matrices. In order to determine the objective focusing correct position, one of the images is steady, such that the corresponding photosensitive matrix generates a constant photocurrent. The other mirror is turned around thus generating a photocurrent corresponding to the projected image depending on the angular mirror position. The two currents generated by the two matrices are amplified and a differential error signal is obtained, determined by the different illumination of the two matrices through the two optical paths. The differential error signal with the smallest level corresponds to a high correlation of the two signals generated by the photosensitive matrices, and therefore to a correctly focused scene. The mobile mirror is interlocked with a potentiometer that generates a signal proportional with the rotation angle (see Figure 4) [12]. The angular scanning of the mobile mirror generates a variable voltage. 

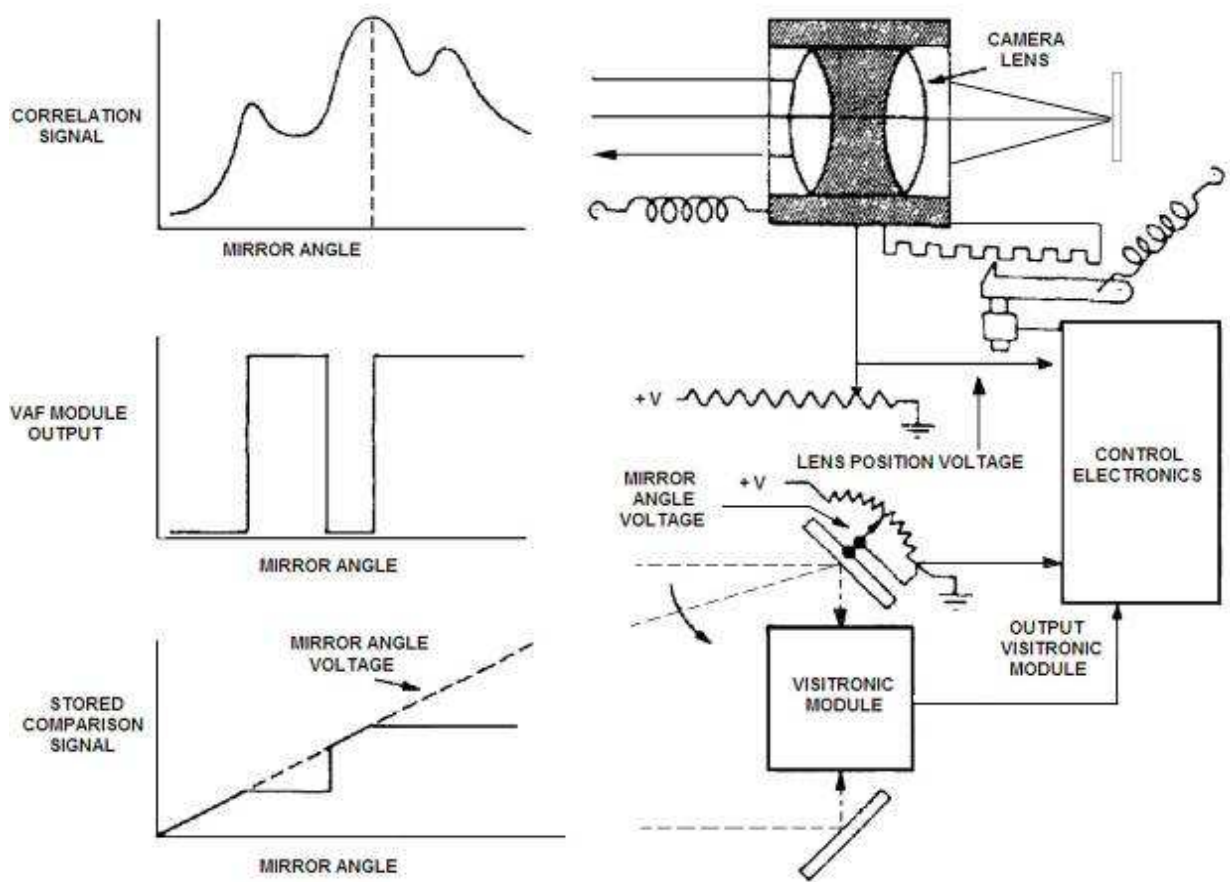

Figure 4. VAF schematic diagram

The voltage given by the potentiometer is electronically sampled and capacitive stored at the moment when the electronic circuit indicates the maximum correlation signal. This voltage is compared with the voltage resulted during the next transition of the mirror position such that, by successive motions, the angular position for optimum focusing is found. The triggering mechanism of the photographic objective is also coupled to a potentiometer, such that the objective motion is stopped at the optimum focusing position. In actual terms, the image analyzer only uses 5 elementary "images", which seems quite primitive as compared to the present systems. Nevertheless, its efficacy was remarkable, being also tested on some orientation robotic systems. At this moment, the technology is visionary; the production of the optical sensor as a photoelectric matrix structure has some technological difficulties. In time, the photocells number increased from $2 \times 5$ to $2 \times 8$ in 1978 , and the production of devices increased to 20,000 units/month!

At the command of AF driving, the mobile mirror starts to explore the scene from a minimum focal distance of $1.1 \mathrm{~m}$ toward the position corresponding to the scene situated at infinity. An indicator visible in a window permits the reading if an "a posteriori control" is desired. In order to charge the apparatus for another scene, the system comes back to the rest position $1.1 \mathrm{~m}$ ) and waits for the analysis of the next image.

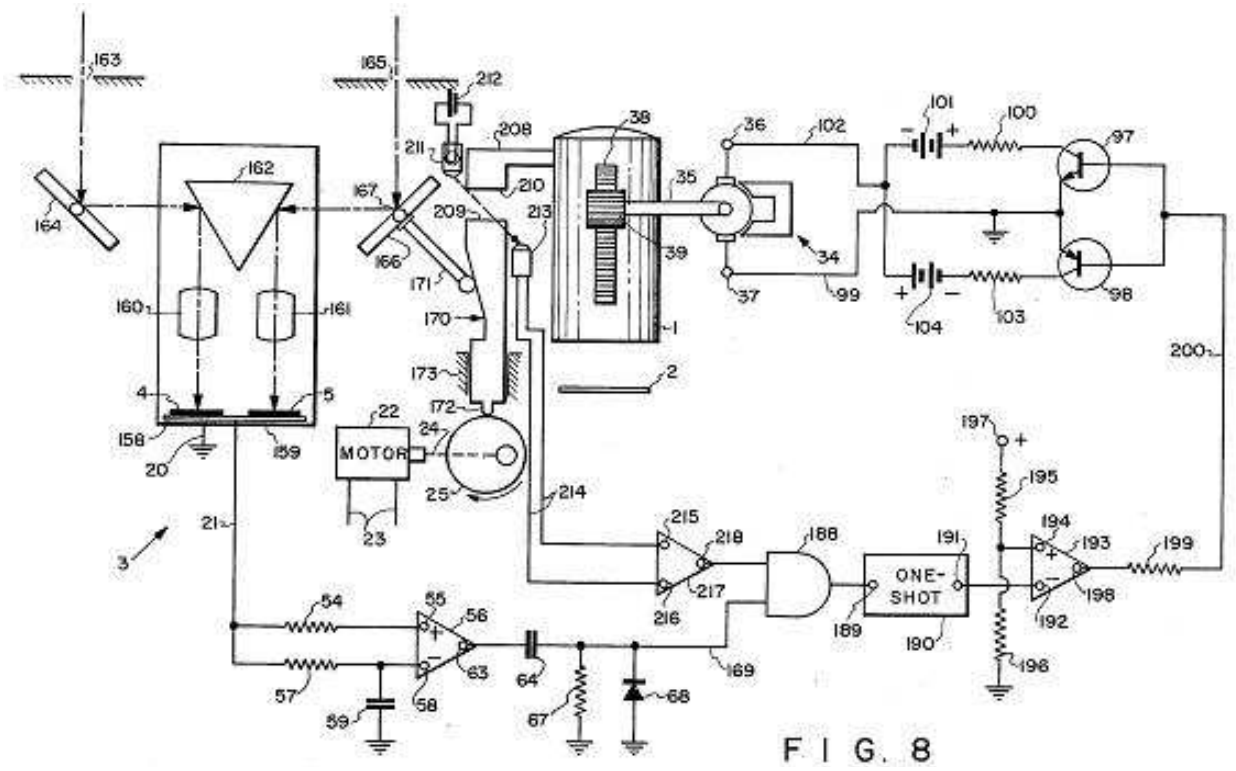

Figure 5. VAF system 
Before filing this patent, Stauffer had filed in 1973 some US patents concerning AF: [13] - [16]; also, in 1976 he was granted the patent US 3,958,117 for a perfected version [18], Figure 5.

Another US Patent No. 4,184,968 from 1980 introduces a compact version of the optical module, the prism and the two mirrors, or lens systems, also including the afferent electronic circuits; the contribution of the electronic technology evolution is obvious in this patent [18].

After the VAF system, Honeywell develops a new autofocusing system called TCL (Through the Camera Lens) based on news inventions by Norman Stauffer, [19] the US Patents 4.185.191 (patent filed in 1978) and 4,384,210 [20]. This TCL system will bring a revolution of reflex cameras, due to the fact that the AF function is accomplished by using a system built-in the camera body. Unlike VAF that used the passive optical triangulation, the new system uses a special objective that includes two antipodal optical sectors that project two images in the focal plane. As these images are different, they can be also called optical signature; at the same time, the TCL uses an optical sensor consisting of a linear matrix $(5 \mathrm{~mm})$ that includes 24 pairs of CCD photocells. The increase of the cell number and, in fact, the increase of their
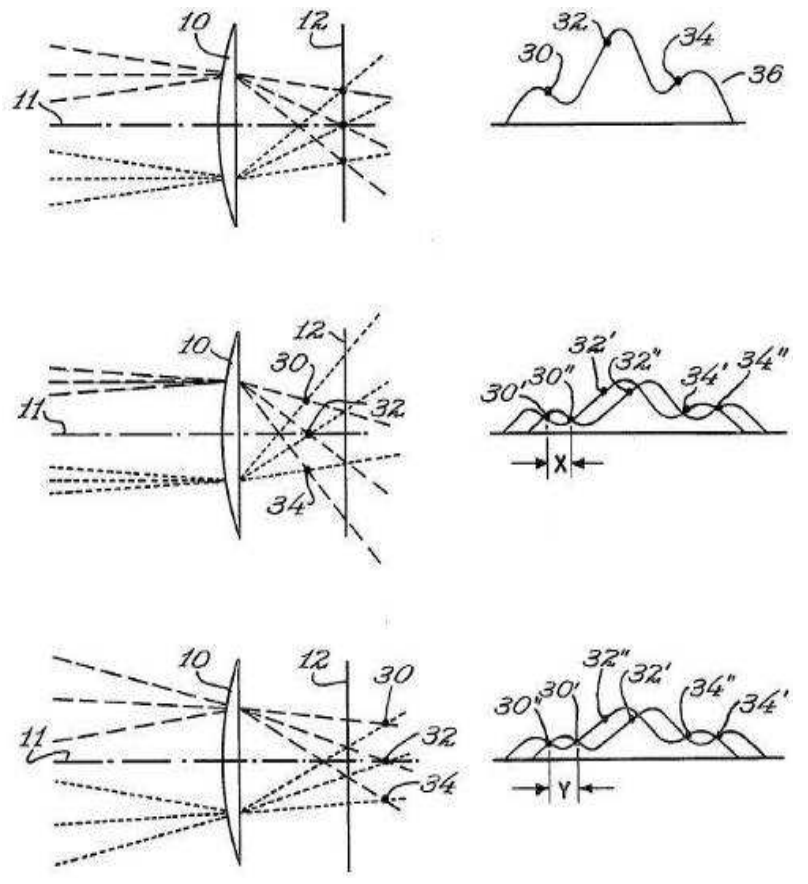

Figure 6. Autofocus - optical operating principle [20]

These modifications of the optical signatures are detected by the linear CCD matrix and transmitted as correction signals to an electric booster to drive the objective. Practically, these signatures are identical and, in the case when the objective is correctly focused, they will overlap each other. When the objective is not focused, the optical signatures will be side shifted, and the image will be blurred. Each CCD photoelement has in front of it a focusing microlens. From the projected image one can obtain 24 samples, each corresponding to a CCD element. In this way, all the 24 CCD

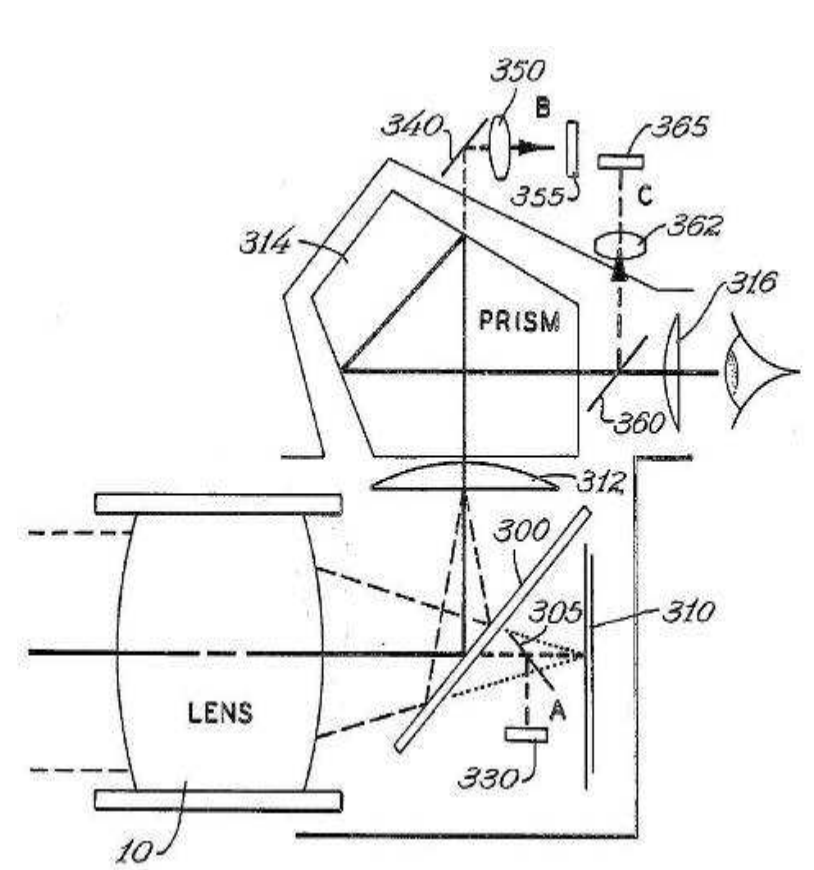

density per unit length by reducing the matrix size amplified the system performances, as the equivalent resolution of the image analyzer also increased. The light beams from these two sectors fall on a series of 24 microlenses installed on the surface of the CCD integrated circuit located in the camera image plane. The image plane is fixed, the photosensitive film being situated here. The technical solutions described in the patent are remarkable, as they open a series of possibilities to the manufacturers of both camera tubes and SLR with AF.

The TLC optical system can be seen in Figure 6; the elements marked with 330, 355 and 362 represent the CCD sensors and their arrangement in various constructive versions. Figure 6 presents three situations:

- three points of an object whose image is correctly focused produce in the image plane of the objective a corresponding optical signature;

- if the object is located at a bigger distance from the objective, the image plane shifts to the objective, and the optical signature of the three points will have a new aspect.

When the object comes closer to the objective, the image plane draws away from the objective, a new optical signature appearing. elements contain the electronic image of the considered image segment, making together an electric potential "relief" which represents the electronic signature. The CMOS integrated circuits provide the amplification, A/D conversion, storage and analysis of the image electronic signatures. By means of dedicated logics, one provides the control of the booster that establishes the sense of shifting the focusing lens or objective position and its fixation in the position corresponding to the optimum focusing. The electronic circuits are supplied by a source of $+5 \mathrm{~V}$ and their consumption is $60 \mathrm{~mW}$. The precision 
of the system consisting of the objective, electronic circuits and micromotor provides the focusing with a deviation of at most $0.05 \mathrm{~mm}$ from the correct image focal plane. As compared to the human eye, its sensitivity and accuracy in establishing the focusing is ten times better.

Soon after, the TLC system was used with different modifications by many manufacturers of video and photo cameras. The first Honeywell patents concerned the passive AF systems; Staufer and Wielwerding patented a series of active systems; these used for focusing an image projected by the subject (Stauffer and Wilwerding, 1981), and patent US 4,317,991 [21].

Other US patents that use both the initial AF principles and their developments are: US 4,207,461 [22], US 4,247,762, [23], US 4,812,640, [24], US 4,474,446, [25] and US 4,593,322 [26].

In 1975 Honeywell gives up the utilization rights for the patents concerning the automatic focusing system, but it exploited its position in a series of international suits.

In 1971, the Nikon Company presented a prototype of automatic focusing apparatus [27] having an objective with the focal length of $80 \mathrm{~mm}$ and an aperture of $\mathrm{f} / 4.5$ (see Figure 7).
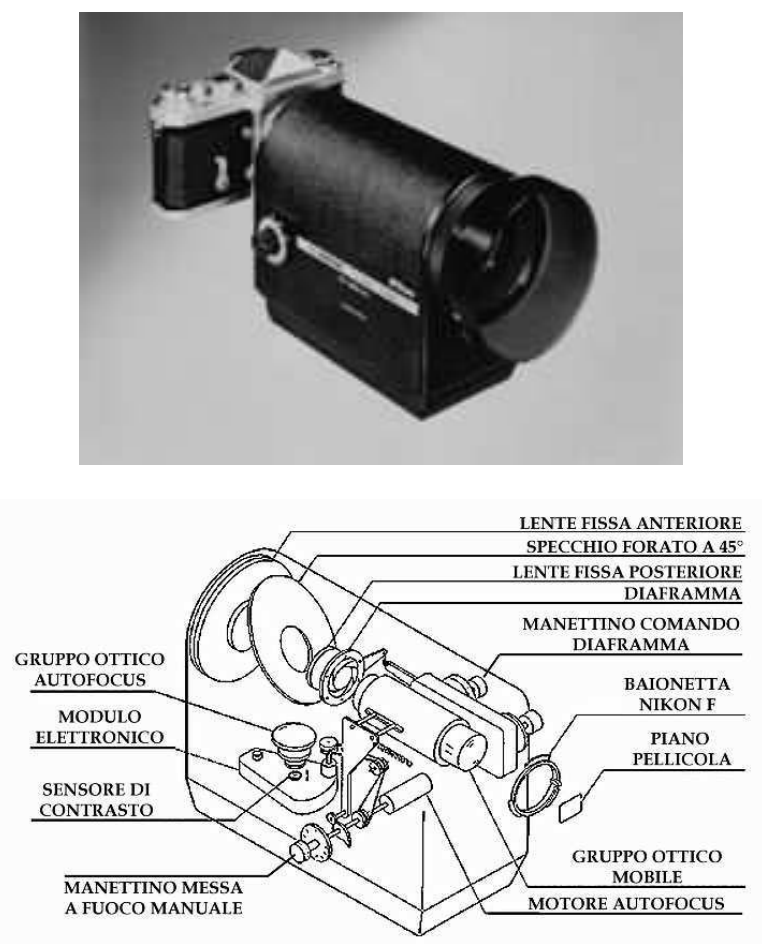

Figure 7. Nikon camera with AF Nikkor 80mm objective, f/4.5

The Nikon Research Laboratories started in 1965 the development of an automatic focusing module for an objective destined to SLR apparatuses, and in April 1971 it launched such an apparatus having the AF function, equipped with a Nikkor $80 \mathrm{~mm}, \mathrm{f} / 45$ objective.

The objective raised a special interest both at the exhibition of Chicago (April 1971) and at Photokina 1972 (September) [28]. The objective was huge for that time: $(289 \times 90 \times 145)$ $\mathrm{mm}$, with an angle of $30^{\circ}$, weighting $2.7 \mathrm{~kg}$, and with focusing from infinity to $1 \mathrm{~m}$.

In 1974, Nikon Corporation introduced a new automatic focusing camera of relatively large size, which made it quite incommode.

At Photokina in 1976 the Leitz Camera AG (Leica) company introduced the prototype photo camera with named Correphot [29] with AF, the result of the researches for the realization and patenting if some automatic focusing technologies and photosensitive matrix sensor destined to automatic focusing. This is the first modern photo camera that uses the image transmitted through the objective for focusing and sharpness detection in a plane equivalent to that where the photo film is located. The apparatus was developed on the structure of Leicaflex SL2.
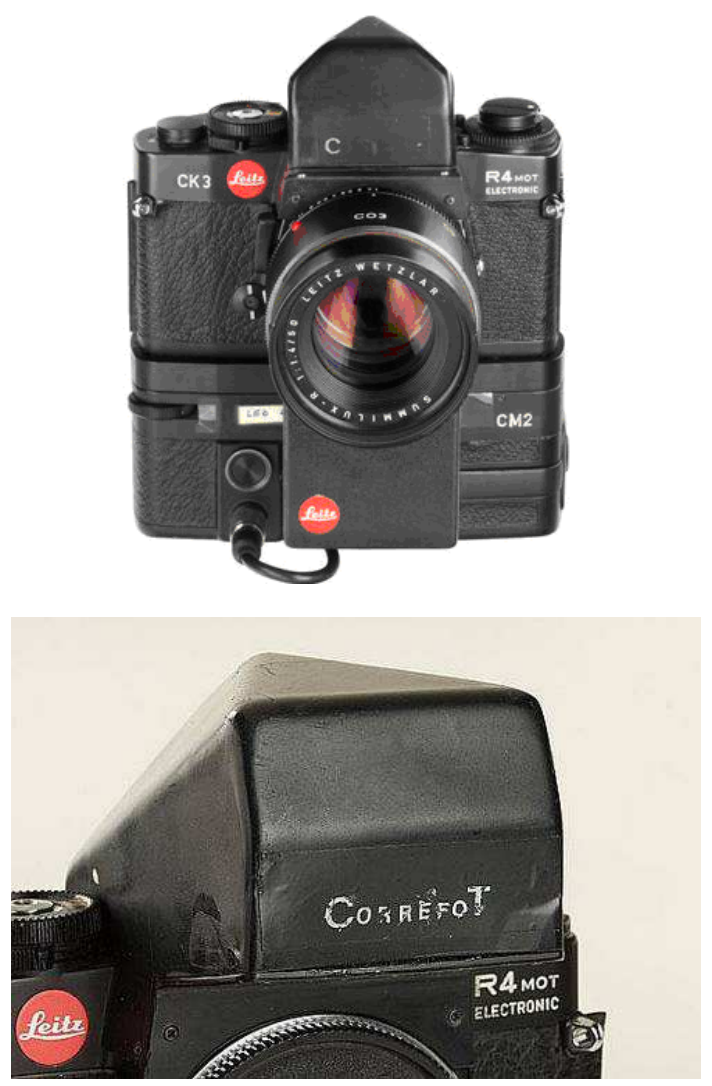

Figure 8. Correfot R4 MOT CK3 prototype

The Correfot name refers to the maximum correlation of the two elementary light beams that pass through the extreme margins of the optical lens block and are generated by an elementary image point of the same subject. The optical system consists of a prismatic network that moves in a plane equivalent with the film image plane; the scene projected through the image of the two opposed halves of the exit pupils of the objective is projected, due to the displacement of the prismatic network, on the surface of two pairs of photocells $\mathrm{AA}$ and $\mathrm{BB}$. These receive the light beams from the two halves corresponding to the exit pupils of the objective as scene images and make an optical "impression". As the result of the refraction on the prismatic network, the two beams emerging through the exit pupils of the objective are focused 
in a plane situated between the network and a lens that disperses the beams on the photoelectric sensors. In Figure 9 the beams are pictured such that to find a facet that deflects them upward. In the drawing below, the prismatic network shifted such that the beams are deflected downward. In each situation, the lens directs the light beam on the pairs of photoelectric sensors connected to the electronic circuits of signal amplification and processing. The three oscillograms represent the signals resulted from the scanning of the two beams across the photoelectric sensors, corresponding to the objective position, focused (in the center) and de-focused in the near plane (left) and remote plane (right). In this way one can determine the direction to which the objective must be shifted depending on the phase difference between the two signals supplied by the photoelectric sensors.

Between 1976- 1982 several versions were realized:

- Leica (SL2) prototype;

- SL2 CK1 with attached control unit for auto-focus);

- R4 MOT prototype with special CORREFOT prism;

- R4 MOT (1978) and R4 MOT C (1980) with CK3 control unit- the most complete camera with auto-focus prototype lens Summilux $1.4 / 50 \mathrm{~mm}$, and CM2 control unit- all these cameras were also used for demonstration during Photokina $1976-1980$

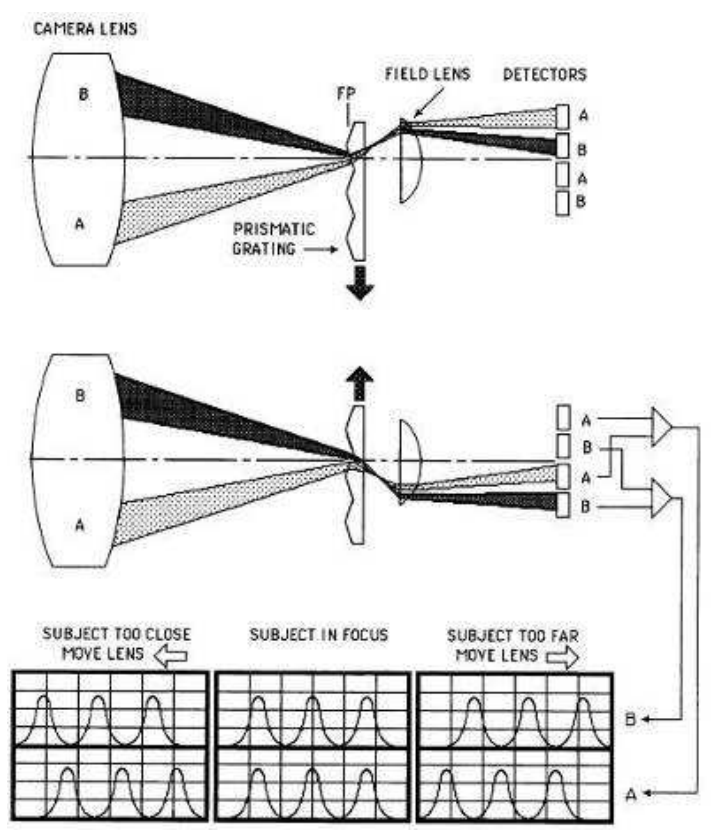

Figure 9. AF Correfot principle

The results of certain researches concerning new proprietary AF technologies were also presented at Photokina 78 [30] with SLR apparatuses having an operational AF.

Also at Photokina'76, Pentax presented a complex autofocus apparatus with zoom (35-70) mm, f/2.8 and an electronic telemeter (Visitronic) that drives the focusing motor. Despite the large dimensions and mass $(1.1 \mathrm{~kg})$, together with other imperfections connected with focusing at low illumination levels and reduced object contrast, the specialists anticipate a future on the market (see Figure 10).

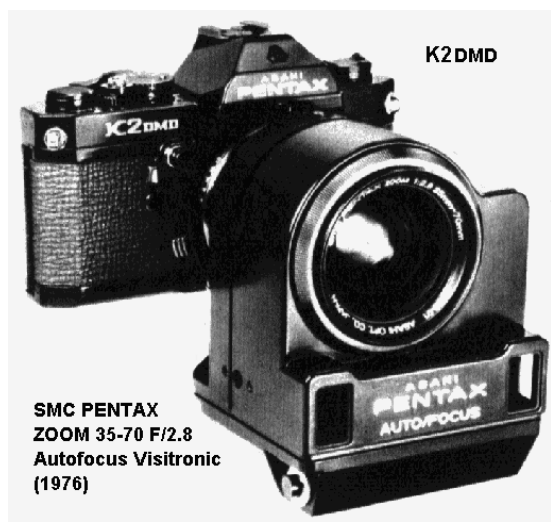

Figure 10. Pentax K2 DMD Autofocus Visitronic

In 1977, Konica launches the first compact mass production apparatus $24 \times 36$ with automatic focusing C35AF, also named "Jasupin".

Launched in November, the camera uses the Visitronic AF, $V A F$ system that was previously developed and produced by Honeywell. In its frontal part the apparatus has the objective, the window for photocell, optical view finder and two identical windows for the VAF system, arranged symmetrically upside, on each part of the manufacturer inscription (Konica). As the AF system operates by analyzing the contrast of two images coming from outside, from the photographed scene, the system is called passive. Given the fact that the VAF method and the electronic telemetry system are especially efficient, they were adopted by other manufacturers too. The process of auto-focusing lasts about 80 milliseconds. The apparatus is equipped with a 2.8 objective of $38 \mathrm{~mm}$; the focusing is not performed continuously, but only on the plateau.

In 1978 they presents an autofocus SLR apparatus destined to the mass production. Later on they produce various versions of pocket autofocus cameras Konica C35 MF (1982) "Jasupin Super" and Konica C35AF (1983); these also use the VAF Hengniweir systems manufactured by Honeywell. This apparatus, of which one million copies were produced and sold, was replaced with a more modern version, without essential modifications, namely C35 AF2 launched at Photokina '80.

At Photokina '78 were introduced some accomplished realizations of compact apparatuses $(35 \mathrm{~s})$, the proliferation of the autofocus, built-in flash models being obvious. All these use the AF Visitronic VAF technology with a built-in infrared source. Among the companies participating with AF cameras we can quote Chinon, Cosina and Fujica.

Chinon launches the $35 \mathrm{~F}$ - $A$ apparatus, a more sophisticated version that uses the Honeywell Visitronic autofocus situated horizontally above the objective. Camera has an objective of $38 \mathrm{~mm}, \mathrm{f} / 2.8$, shutter (1/8- 1/500), super-exposure warning, flash exposure from $1.2 \mathrm{~m}$ to $4.5 \mathrm{~m}$. Another apparatus introduced is 35 F-MA Infrafocus, scheduled for production in 1979.

Afterwards, the company develops the production with the versions Chinon 35FA Super and Chinon 35 FA II; both use IR triangulation, the AF modulus being positioned vertically on 
the right side of the objective.

Chinon develops new versions, with an original and attractive design that use the same IR AF system located horizontally above the objective, a system that can be noticed at Chinon 35F-MA Infrafocus.

Cosina Co. Ltd of Japan launches the apparatus Cosina AF 35 Autofocus Flashmatic EE; unlike the previous versions, it permits to avoid the automatic focusing in favour of the manual one. It was followed by Presenta and $C X 70$ versions.

Fujica has introduced three cameras that also use the Honeywell Visitronic focusing systems. The most complex is Flash Fujica Auto Focus, which resembles Chinon 35 F-A (indication in view-finder of the manner in which the autofocus works), to which some distinctive elements are added: autofocus lock, with the shutter release button, a "beam sensor" for the camera switched to flash mode. The beam sensor transmits a light beam in the direction of the subject to increase the level of his illumination and make the AF work more correctly.

Yashika introduces the Auto Focus camera with an objective of $38 \mathrm{~mm}, \mathrm{f} / 2.8$, aperture $\mathrm{f} / 2.8-\mathrm{f} / 16$, consisting of four elements of three lenses for each group, shutter speed 1/500, light meter - CdS, ISO 25- 500, flash- built in only, indicator showing the AF activation (shows what AF locks on), light signals (LED) for reduced illumination.

Also in 1978 Polaroid launches the SLR OneStep Sonar camera (accomplished starting from its first model $S X-70$ ), this being the company first apparatus with $\mathrm{AF}$ available for users. The newness introduced by them consists in the utilization of a TOF (Time of Flight) method for distance measurement, based on a patent by Biber Conrad. It his patent [31]. (filed in 1968 and patented in 1970) Figure 11, Biber uses an US telemeter consisting of two transducers, one for emission and another for reception. The propagation duration modulates an impulse of variable duration which controls a booster that shifts the objective in the position corresponding to focusing. The Polaroid telemetric system uses a single ultra-acoustic electrostatic capacitive transducer with gilded polyester foil (playing the part of emitter and receiver), supplied at a high voltage, and a digital electronic module. It is simple, quite precise and relatively cheap to provide a mass production. It was also used in other applications, such as robots, alarm and orientation systems [32].

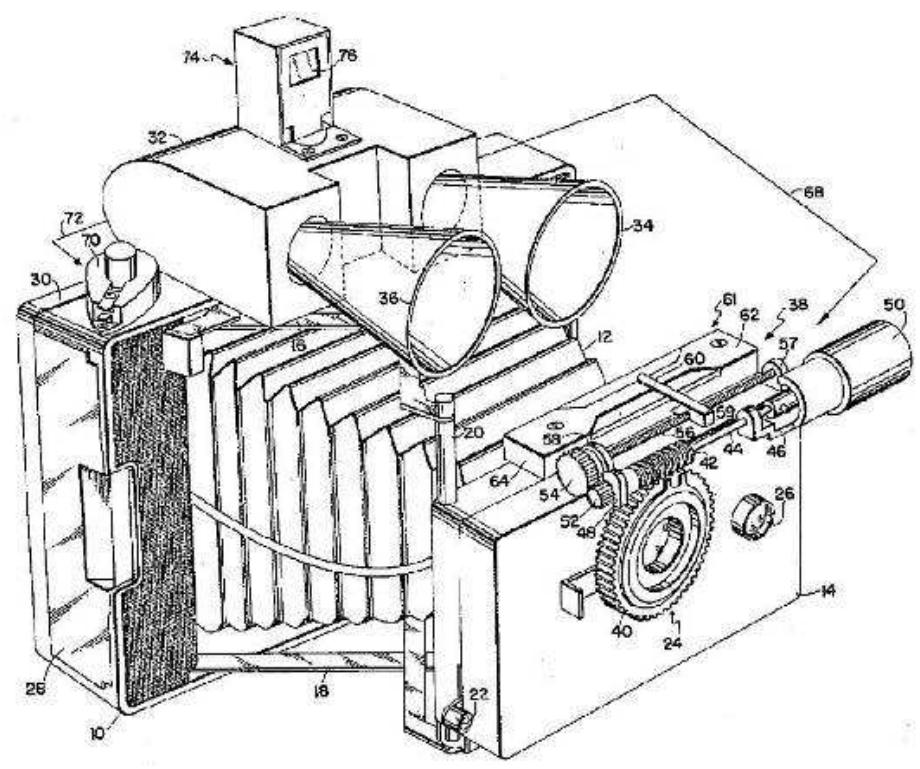

Figure 11. Rangefinding and focusing system for photographic cameras

Later on, in 1982, they launch an AF apparatus type "SLR 680 Autofocus Land Camera (aperture range from $\mathrm{f} / 8$ to $\mathrm{f} / 22$, element $116 \mathrm{~mm} \mathrm{f} / 8$ glass lens with a minimum focal length of 10.4 inches). These apparatuses use the sonar specific technology to control the focusing; the ultrasonic system is also named "proactive". A shortcoming appears when the photographed object does not reflect and absorb the ultrasounds or when one takes a photo to an image reflected by a mirror, a window or a polished surface. The AF process is a little faster: $60 \mathrm{~ms}$. Polaroid has invented a series of new devices, with applications especially in automatic focusing, but also in robotics [33], orientation and range finding for movie camera [34], Figure 12.

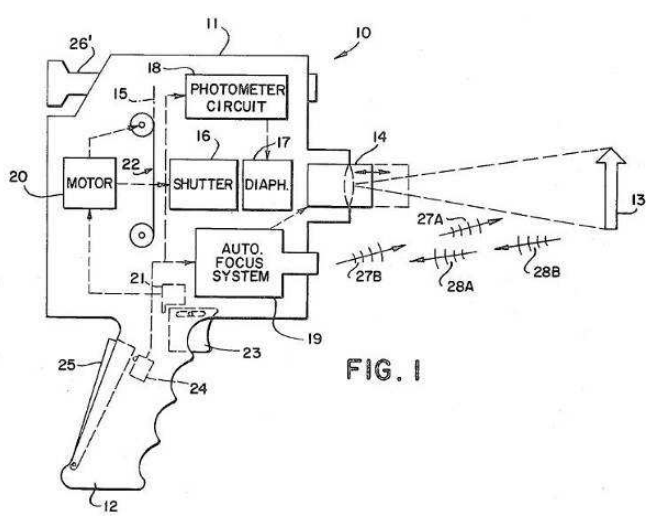

Figure 12. Autofocus movie camera 
In 1978 Sankyo uses the VAF Visitronic module for image focusing in the VAF Electronic System Visitronic Auto Focus ES 44 XL AF camera, Figure 13.

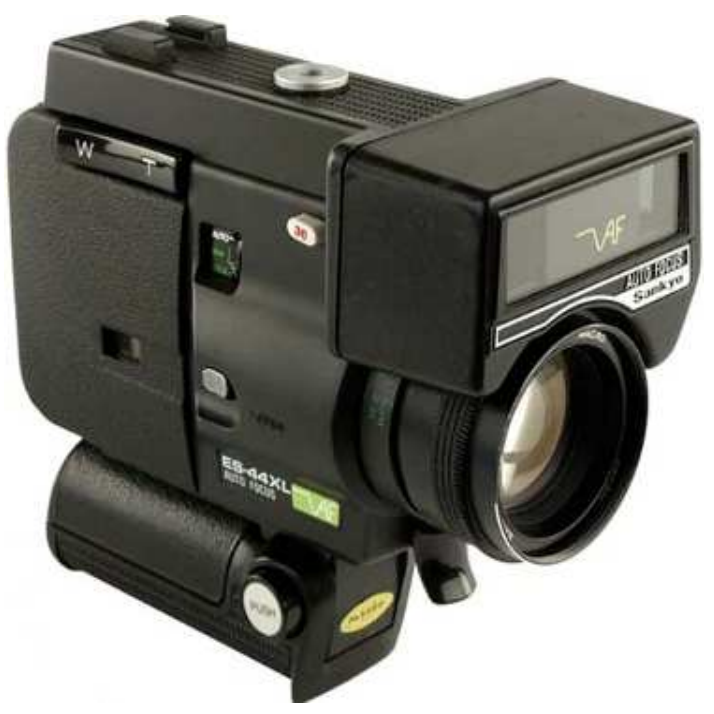

Figure 13. Movie camera ES $44 X L A F$

1979 - Canon develops and launches on the market, 18 months later (in November 1979) than Konica- C35AF, an $A F$ $35 M$ Sure Shot, Figure 14, apparatus, being the first "compact-point and shot camera" that uses an active system with IR radiation, SST- Solid State Triangulation, that becomes popular, such that afterwards this principle was assimilated by other manufacturers too.

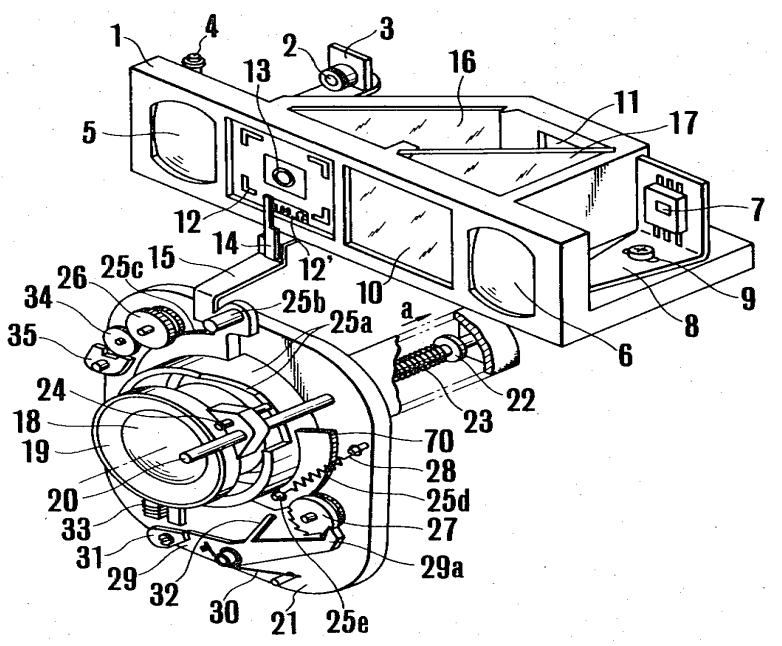

Figure 14. Autofocus camera

The system, patented in 1983, advantages and disadvantages are similar to those of the ultrasonic system, but it is simpler and more compact. Figure 14 presents principle [35] of SST.

Also in 1979, Minolta produces the compact apparatus 35 mm, with AF, Minolta Hi-Matic AF.

In 1980, as the result of the market success of the AF system utilization, Canon and Ricoh produce standard objectives (35-70)/4AF and 50/2AF respectively, fitted with built-in AF. These objectives are mainly destined to SLR cameras. They are also known under the name of external AF; these were quite bulky, because the supply batteries and the driving motor were introduced in a case attached to the cylindrical objective.

In 1980 at Photokina the Ricoh Company introduces an apparatus fitted with a built-in AF system - the Visitronic module. The reflex viewfinder permits to appreciate the gap between the AF and manual focusing. The objective is of 50 mm standard type. In 1983 Ricoh patented an "automatic focusing position detection apparatus of the double image over lapping type" [36].

Canon also demonstrates a camera with Visitronic AF system having a modified design, adapted to the photo camera, with FD 35-70 mm, f/4 objective (launched on the market in 1981). This is the first apparatus with interchangeable objectives. The Visitronic module is placed tilted, to permit the access to controls.

Konica introduces the AF 514 /4X-S camera that uses VAF Visitronic SST (Solid State Triangulation).

Mamiya produces a compact apparatus, $135 \mathrm{AF}$ with built-in flash and active AF, using IR triangulation

Later on, in the years 81 's- 84 's, the company develops AF systems destined to SLR cameras: Patents 4,381,523 [37] and $4,423,935$ [38], with an increased precision obtained by avoiding the parallax errors between the objectives and the IR telemeter, adopting the TLC system introduced by Honeywell.

In July 1981, Canon introduces the new active focusing system called SST (Solid State Triangulation) that also uses the IR triangulation to a pocket $A F 35 M L$ camera with a new objective f/1.9. Afterwards, during the years 1983-1986, based on some patents [39], they develop this apparatus under the name $A F 35 M I I$.

Based on the prototype of 1979 , several versions were produced, known under different names in the USA, Europe and Japan.

1981- Canon Super Sure Shot AF35 ML Autoboy Super

1983- Canon (New) Sure Shot AF35 M II Autoboy 2

1985- Canon Sprint/Jet (AF35J)/Autoboy LITE

1986- Canon Sure Shot Supreme/Top Shot/ Autoboy 3

Canon Sure Shot Tele/Top Twin/Autoboy Tele.

1981 - Olympus (after the attempt from Photokina '78) launches their first $C$ - $A F$ apparatus with active AF that uses the infrared radiation to control the focusing. This is also its first apparatus with built in flash produced until 1986. The utilized AF system does not belong to them, but is imported from another manufacturer.

The apparatus was not delivered all over the world; it might have been a pilot technological attempt to mark a change in the photo cameras technology. They combine in the same apparatus a mass rangefinder, together with the autofocus technology, instead of the telemeter, two techniques completely apart from each other in term of technology. The objective is $\mathrm{f} / 2.8$, with programmed exposure. Subsequently, Olympus developed its production with the versions:

AFI Picasso (1983), the name deriving from Japanese "pika" which means "flash"; this is the first camera that uses lithium batteries.

Nurepika (Wet Flash), the first completely protected AF 
compact camera, waterproof, sandproof and dustproof. AF-1 Twin Quartzdate (1988) - also a waterproof camera

with two built in objectives, one of $35 \mathrm{~mm}$ and another of 70 $\mathrm{mm}$, switchable through a button.

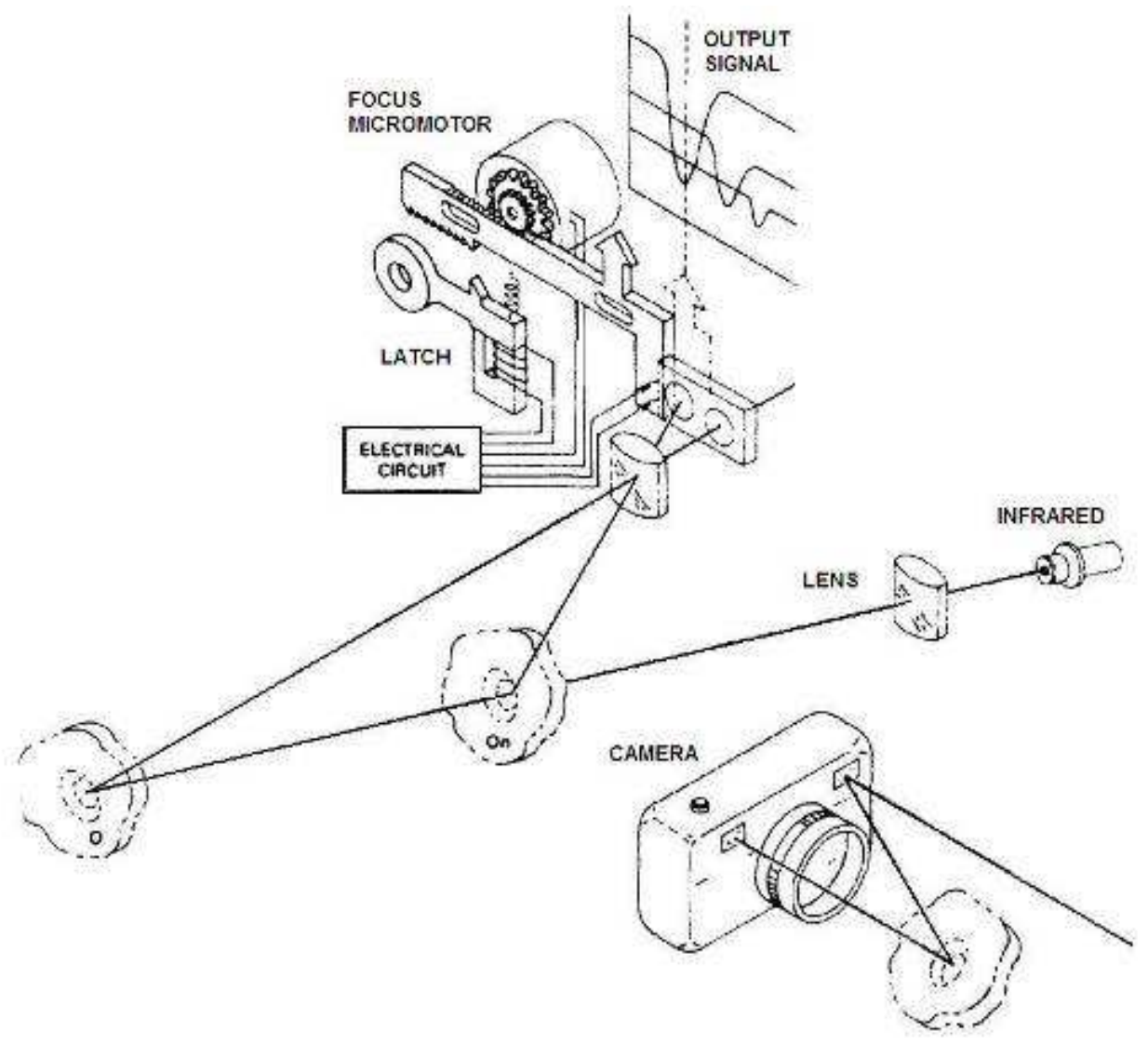

Figure 15. Principle of the Focus Control Module AutoFocus Pentax

At the end of the 70's, Pentax Corporation develops an electronic and electro-mechanic system for automatic focusing. The system (see Figure 15) named FCM-AF; Focus Control Module [40] was used at the first compact AF camera, Pentax Sport. This is an active triangulation system that uses the modulated infrared radiation with the frequency of $4 \mathrm{kHz}$. The action range is $(0.75-4.0) \mathrm{m}$, with a number of 8 steps, the precision being of one step only; the driving time is $70 \mathrm{~ms}$.

Given its performances, the system was also used in other applications related to robots control.

1981- Asahi realizes the first Pentax MF-F apparatus, the first really reflex camera destined to be sold to a wide public, SLR $35 \mathrm{~mm}$ equipped with AF, having a dedicated objective fitted with control motor built-in the camera body. The AF sensor and its supply source, located in camera body, communicate through electric contacts located on the bayonet, with the objective that includes the control motor and its own supply. Making use of a classical optics, the MF-F apparatus also offers an assisted focusing, accessible through the LED diodes built in the view finder.

Asahi has made outstanding contributions in AF but also in related system, for example the patent "Automatic focus camera with interchangeable lens" [41]. The camera uses the contrast detection by means of three linear CCD sensors, the information about focusing being calculated by a microcomputer and indicated in the view finder.
In 1982, March, Canon was introduced Canon AL-1 Quick Focus camera which is the largest camera manufacturer in the world.

1982- Olympus presents at Photokina the OM 30 cameras with $35-70 \mathrm{~mm} \mathrm{f} / 4$ objective. It seems that this is the first photo camera that uses a sensor which analyses the phase shift (sometimes called phase contrast - not to be mistaken for the systems that analyze the image contrast). The light beam is sent from the objective through an optical system that divides the beam for the two CCD optical sensors through which the focusing is detected; the image is clear when each half-beam is focused in the center of each CCD optical sensor.

If there is a phase shift between the images, this is converted by the optical sensors into an electric signal that commands the objective position by means of a motor. Even if at that moment, 1982, this method faced some problems concerning the electronic, optical and mechanical technologies, it came into focus and soon was assimilated and developed by other companies.

Contax presents at the same fair of 1982, a SLR $35 \mathrm{~mm}$ apparatus, developed on the basis of Contax $137 \mathrm{MD}$ camera, to which it attaches a case containing the AF module. The command and the motion for focusing are transmitted to the objective by the motor by the means of a fork located in the bayonet holder. Even if Contax came with an innovating technology, it did not use it for a mass production; therefore it 
was taken over by Minolta, Nikon and Pentax too. Contax develops later (1996) a new AF technology named "Automatic Back Focusing" and introduces two innovations that increase the precision of the objective control

- ceramic guides to command the engine and the motion of the film plane back and forth for focusing;

- piezoceramic ultrasonic motor that provides a high precision of the back and forth motion control.

The focusing modification from the minimum distance to infinity is performed within a $450 \mathrm{~ms}$ interval. Contax uses in the new system some tents of patents.

The AX model introduced in 1996 is the only camera that corrects the focusing by "moving the image plane". This innovation has a special advantage, mainly in microphotography, since it permits to obtain images with a ration exceeding 1:1, without introducing a bellows or ring; this is a complex camera equipped with three Central Processing Units (CPU) that control all the camera functions.

1982-Minolta produces a compact $35 \mathrm{~mm}$ apparatus fitted with a motor, namely Minolta Hi-Matic AF2 Motor.

The AF system was introduced relatively late, even if there were researches concerning $\mathrm{AF}$ in the patents literature. At the end of the 70's, Minolta develops patents a series of technical solutions concerning the utilization of photoelectric sensors for focusing; afterwards the first patents appear using IR active optical triangulation [42], [43], [44], [45].

1983- Minolta launches the X 500 (SLR, TTL) AF apparatus that uses the "phase detection focusing"; this has the motor and the AF sensor built in the camera body.

Canon patented in 1981 an AF system that uses the IR triangulation [46].

Even if their principles are similar, the technical solutions patented by Canon, Minolta and other manufacturers differ by original elements, adapted to technological possibilities: utilization of modulated or un-modulated radiation, objective's controls, photosensitive elements, IR filters, adjustment and calibration elements, etc. This technique was modernized and assimilated more and more by manufacturers who introduced optical and adjustment elements made of plastic materials. Starting with this moment, self-tapping screws and glues are used for assemblage. The promotion of these cameras was backed up by the special qualities of the plastic materials, especially their mechanical and dimensional stability, as well as by the facilities related to mass production through injection of some mechanical elements, their fast assemblage and integration at a wide scale, which permitted the utilization of three processors each.

In 1983 Canon launches a new version of the AF $35 \mathrm{M}$ camera, called "Sure Shot" in America, "AF35MII" in Europe and "Autoboy" in Japan.

At the same time, in March 1983 they launches the T50 camera that is produced until December 1989; the apparatus was followed by the series T60 (1991- 1992), T70 (1984), T80 (1985-1986), T90 (1986).

In 1983, the Nikon launched the photo camera SLR Nikon $F 3 A F$, having the automatic focusing function executed by means of two lenses from the AF Nikkor objective- $(80 \mathrm{~mm}$ and $f / 2.8$ ) and $A F-200 \mathrm{~mm}, \mathrm{f} / 3.5$. This was the first photo camera with AF function produced by Nikon. The AF sensor comes from Honeywell, proprietary of the AF patents.

1985 - Canon launches in April (two months after Minolta 7000) its first apparatus T80, SLR, AF $35 \mathrm{~mm}$ and produces it for only one year, until April 1986. This belongs to the series of T camera with FD holder. A CCD linear matrix was used for AF sensor. The AF lenses from T80 were called AC and they have a FD holder with contacts for electric signals transmission. It can be fitted with three such lenses: AC 50 $\mathrm{mm}, \mathrm{f} / 1.8$, AC $35-70 \mathrm{~mm}, \mathrm{f} / 3.5-4.5$ and AC 75-200 mm f/4.5.

At Photokina 1986, Minolta launches the Maxxum - 7000 camera, this being the first SLR with built-in AF function, the driving motor being introduced in the camera body. The apparatus was a market success, having also a beautiful architecture that inspired other companies, like Nikon and Pentax. It is also known under the names Maxxum, Dynax, Alpha 7000, depending on the trading place: USA, Europe or Japan.

In 1986, the BelOMO of Minsk, URSS, produces the first soviet camera with automatic focusing. The BelOMO Company developed from "Minsk Mechanica Factory" established in 1957 that manufactured optical glass and photographic cameras which were afterwards produced by the GOMZ Company. In 1971 the factory converted in BelOMO (Bielorussian Optical and Mechanical Association) Company, with headquarter in Minsk- Bielorussia. It produced only one photo camera (half frame) named Chaika.

The Elikon Autofocus apparatus, with photographic film of 24x36, objective Industar 95, aperture 2.7/38, shutter electronically controlled in steps $(1 / 8 \mathrm{~s}-1 / 500 \mathrm{~s})$, parallax correction in viewfinder, electronically controlled flash, and plastic case; the film is loaded automatically and the focusing range is from $1.1 \mathrm{~m}$ to infinity. Except for the AF function, the camera is identical with Elikon $35 \mathrm{C}$. It was produced in three versions named also Elikon. The prototype version is an extremely rare camera. The common version, PN 5710, has the camera body slightly changed and new signs on the camera; on objective appears the inscription DIN/GOST concerning the film sensitivity. The PB 5715 version is identical with PB 7510, still wearing the inscription ISO/GOST. The upper camera part is slightly modified, and the flash indicator is moved.

The export version of the PB 7620 camera is identical with PB 5715, but the flash indicator is marked with Roman characters, and not with Cyrillic letters.

In 1988 Vivitar Corporation launched an AF camera that uses the TLC Honeywell technology. The camera has two AF objectives, one with $\mathrm{f} / 3.5-\mathrm{f} / 22(28-70 \mathrm{~mm})$, and the other with $\mathrm{f} / 4.5-\mathrm{f} / 22(75-200 \mathrm{~mm})$. Each objective offers two operating modes: "single mode" in which the lenses search and fix the sharpest focusing in a single point, and the other in which the focusing is continuous, monitoring the pursued subject. The focal length is of $1 \mathrm{~m}$ in the normal mode, and in the macro mode it is $0.35 \mathrm{~mm}$.

Figure 16 presents a synthesis of the main AF techniques. The human view is based on the contrast analysis of the image 
formed on retina.
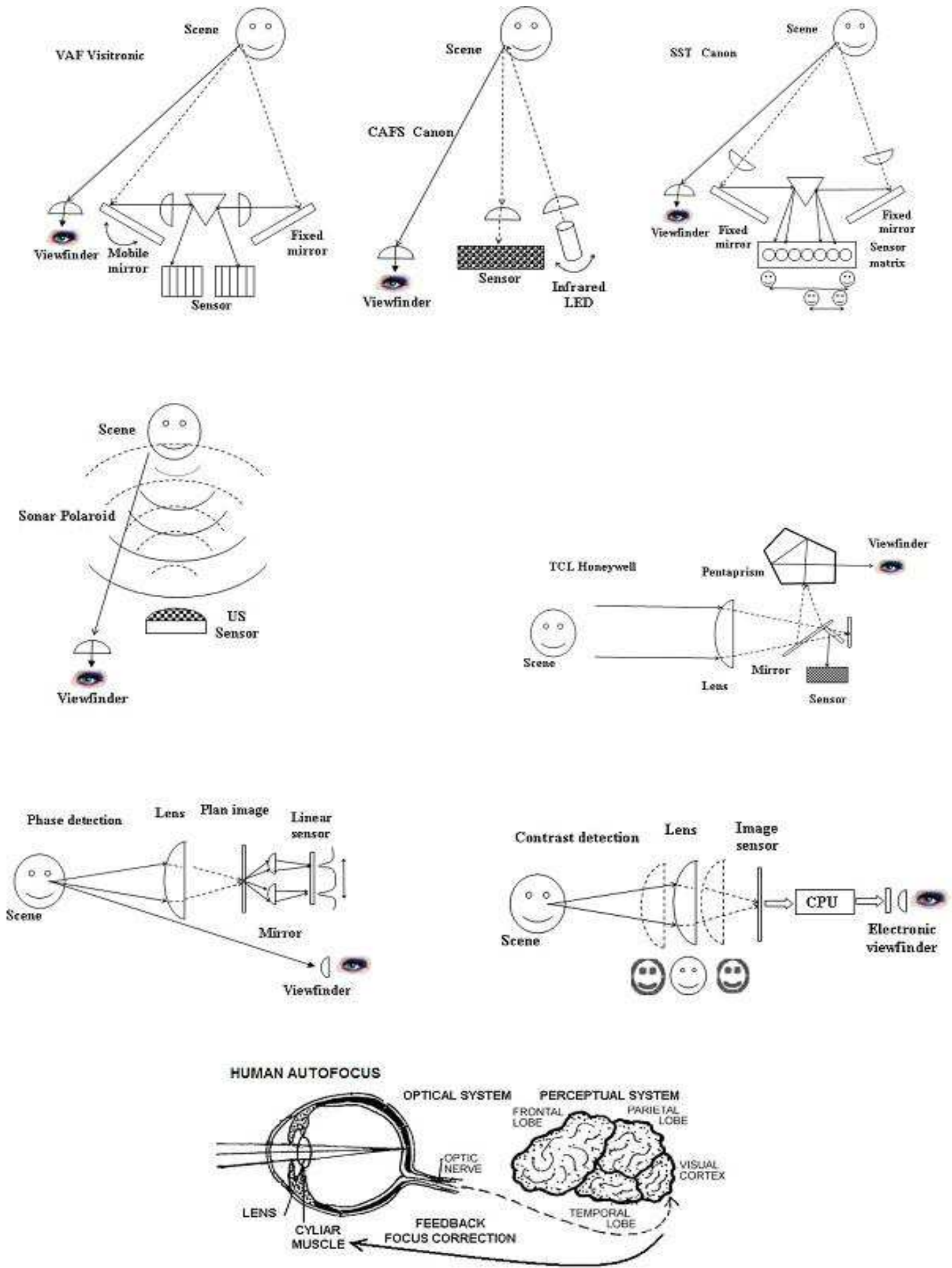

Figure 16. Synthesis of AF systems 
After these years, the evolution of the photo cameras turned toward their fitting with performing image processors, increasing the resolution, the focusing speed, the image storage capacity, the photo cameras also including macro-photographing option, shooting, operation in UV and IR ranges, complex image processing, etc.

Table 1. Evolution of the autofocus systems

\begin{tabular}{|c|c|c|c|}
\hline Year & Firm & Camera name & Principle \\
\hline 1963 & Canon & Prototype Photokina 63 & UN \\
\hline 1965 & Baltag & No name, Patent RO 44,277 & $\mathrm{CD}$ \\
\hline 1971 & Nikon & Prototype & UN \\
\hline 1972 & Nikon & Lens Nikkor 80 & UN \\
\hline 1974 & Nikon & $\mathrm{UN}$ & UN \\
\hline 1975 & Hengniweier & AF telemetry devices & T, VAF \\
\hline 1976 & Leitz Camera & Correphot SLR CK1 & PDF \\
\hline 1976 & Pentax & K2 DMD & VAF \\
\hline 1977 & Konica & $\mathrm{C} 35 \mathrm{AF}$ & VAF \\
\hline 1978 & Honeywell & 24 lens, telemetry & TCL \\
\hline 1978 & Konica & $\mathrm{UN}$ & UN \\
\hline 1978 & Chinon & $35 \mathrm{~F}-\mathrm{A}$ & VAF \\
\hline 1978 & Chinon & 35 F-MA Infrafocus & IR \\
\hline 1979 & Cosina & CX 70 & IR \\
\hline 1978 & Fujica & Flash Fujica AF & IR \\
\hline 1978 & Fujica & Flash Fujica S & IR \\
\hline 1978 & Yashica & Auto Focus Motor & IR \\
\hline 1978 & Polaroid & SLR One Step Sonar & US \\
\hline 1978 & Sankyo & ES-44XL video camera & VAF \\
\hline 1979 & Canon & AF $35 \mathrm{M}$ & IR \\
\hline 1980 & Mamiya & Mamiya $135 \mathrm{AF}$ & IR \\
\hline 1980 & Ricoh & Ricoh $50 \mathrm{~mm}$ & VAF \\
\hline 1980 & Canon & Canon Visitronic & VAF \\
\hline 1980 & Konica & C35 AF 2 & VAF \\
\hline 1980 & Sankyo & XL-320 video camera & VAF \\
\hline 1980 & Canon & AF514XLS C video camera & VAF \\
\hline 1981 & Honeywell & Patent US 4,247,762 & $\mathrm{CD}$ \\
\hline 1981 & Canon & AF $35 \mathrm{ML}$ & SST, IR \\
\hline 1981 & Olympus & C-AF & IR \\
\hline 1981 & Asahi & Pentax MF-F & PDF \\
\hline 1982 & Pentax & Pentax PCF 35 AF & IR \\
\hline 1982 & Canon & AL-1 & QF \\
\hline 1982 & Olympus & OM 30 & PDF \\
\hline 1982 & Polaroid & SLR 680 & US \\
\hline 1982 & Konica & C35 MF Jasupin Super & VAF \\
\hline 1983 & Minolta & X 500 & PDF \\
\hline 1984 & Canon & AF35MII & IR \\
\hline 1985 & Canon & T80 & PDF \\
\hline 1985 & Minolta & Maxxum, Alpha 7000 & $?$ \\
\hline 1986 & BelOMO & Elikon AF & $\mathrm{T}$ \\
\hline 1988 & Vivitar & Vivitar & TCL \\
\hline 1996 & Contax & $\mathrm{AX}$ & $\mathrm{ABF}, \mathrm{MIP}$ \\
\hline
\end{tabular}

$A B F$ - Automatic Back Focusing; $C A F S$ - Contrast Angle Focusing System; CD -Contrast Detection; IR - Infra Red; $M I P$ - Moving the Image Plane; $P D F$ - Phase Detection Focusing; $Q F$ - Quick Focus; SST - Solid State Triangulation; $T$ - Triangulation; $T C L$ - Through Camera Lens; $U N$ Unknown; US - UltraSound; VAF - Visitronic Auto Focus.

\section{Active and Passive AF - A Comparison}

The remarkable technological progresses generated the utilization of the AF systems in the photo and video cameras. The two systems: phase detection and contrast detection, are applied in most of the SLR cameras.
The phase detection, sometimes called also "phase contrast", needs a relatively complex optical system, due to the existence of an additional optical module that works like a dividing lens (two separate sectors of the same lens) through which two images are photographed apart from each other, containing different information about the scene transmitted through the objective. By measuring the distance between the two images, one can get information about the objective position with respect to the accurate image plane. If the distance between the two images is smaller (or bigger) than an optimum value, it means that the system is out of focus; in this situations, the objective focal plane is either in front of (anterior focusing) or behind (rear focusing) the image plane. Depending on this information gathered by the processor from the linear sensor on which the two images are projected, one can establish the sense and the value of the objective or focus lens shift. This system is fast, as it uses a small number of steps for focusing.

Contrast detection operates on the basis of a simple principle resulted from the optics laws, namely that an image is clear when its contrast is optimum. Therefore, this system must establish the image quality by analyzing the contrast, and the information must be transmitted to the objective to establish its position. In the first moment of the analysis, the system knows neither the image quality (if a correction must be performed), nor the direction to which the correction needs to be made or the value of this correction. Therefore it needs to shift the objective, to establish the necessity of a correction and then to establish if the chosen direction is the right one or it has to be changed. This is a process occurring step by step until the optimum image is determined; therefore, it needs a longer duration than for phase detection. An advantage is still the absence of the additional optical module and of the phase detection optical sensor, as the image analysis is performed by extracting the elements of interest from the electronic image of the scene.

Predictive AF. AF is useful since it disengages the operator when he is focused on a certain element or subject of the scene, tracking the subject position through the view finder. Yet, if the subject is moving fast and often unpredictably, the moment when releasing the trigger is quite difficult to establish, a special ability being necessary or else the image is unclear, in motion and most times without an artistic effect. There is an option named Continuous-servo $A F(A F-C)$ that takes up this shortcoming, but not completely.

This problem is solved by software, namely by using a "system of predictable tracking" that applies special algorithms that provide the optimum prognosis of the subject position at the moment of triggering, when the image sensor is seizing the image. Its operation is based on the detection of the subject speed when he moves in the scene, on permanent adjustment of image focusing in the image sensor plane, as well as on establishing the optimum moment for seizing pictures (Nikon/Technology / Predictive Focus Tracking System).

According to some analyses, the contrast detection provides 
a better image quality than the phase detection; the focusing errors with phase detection are mainly due to mechanic causes and when the objective is in strong light and has a relatively small F (1.4).

Hybrid autofocus consists in simultaneous utilization of the information received from the image sensor, information concerning both the contrast and phase difference.

The autofocus presents obvious advantages, but in certain situations it restrains the operator intentions, especially when the operators wish to perform certain artistic effects by changing the focal plane of the scene. A major disadvantage appears at the objectives fitted with zoom. The zoom effect consists in modifying the angle (and therefore the dimension) under which the subject is seen when focusing. The solution was to introduce an additional CPU control that checks-up and correct the zoom when AF is acting on the image of an object on the scene. This function introduced especially in the cineor TV cameras, is named CAFS (Constant Angle Focusing System).

\section{Description of the First Patent About Contrast Analysis Image}

During the 60's, the TV cameras were not fitted with autofocus systems. Some of them had an analogue instrument that indicated the high frequencies content corresponding to image sharpness. The technical solutions reported in the patent literature could not be applied, since they complicated the camera tube system. The application of the autofocus in photography was also impossible, given the technological level at that time. The solution of processing an image element, a segment of the TV signal line, permitted to interpret the video signal and to make decisions concerning the optic system control such that to produce a sharp image [47]. Therefore this system behaves like an extreme adjustment loop. We shall present in the following the description of the patent and the block diagram of the proprietary solution (see Figure 17).

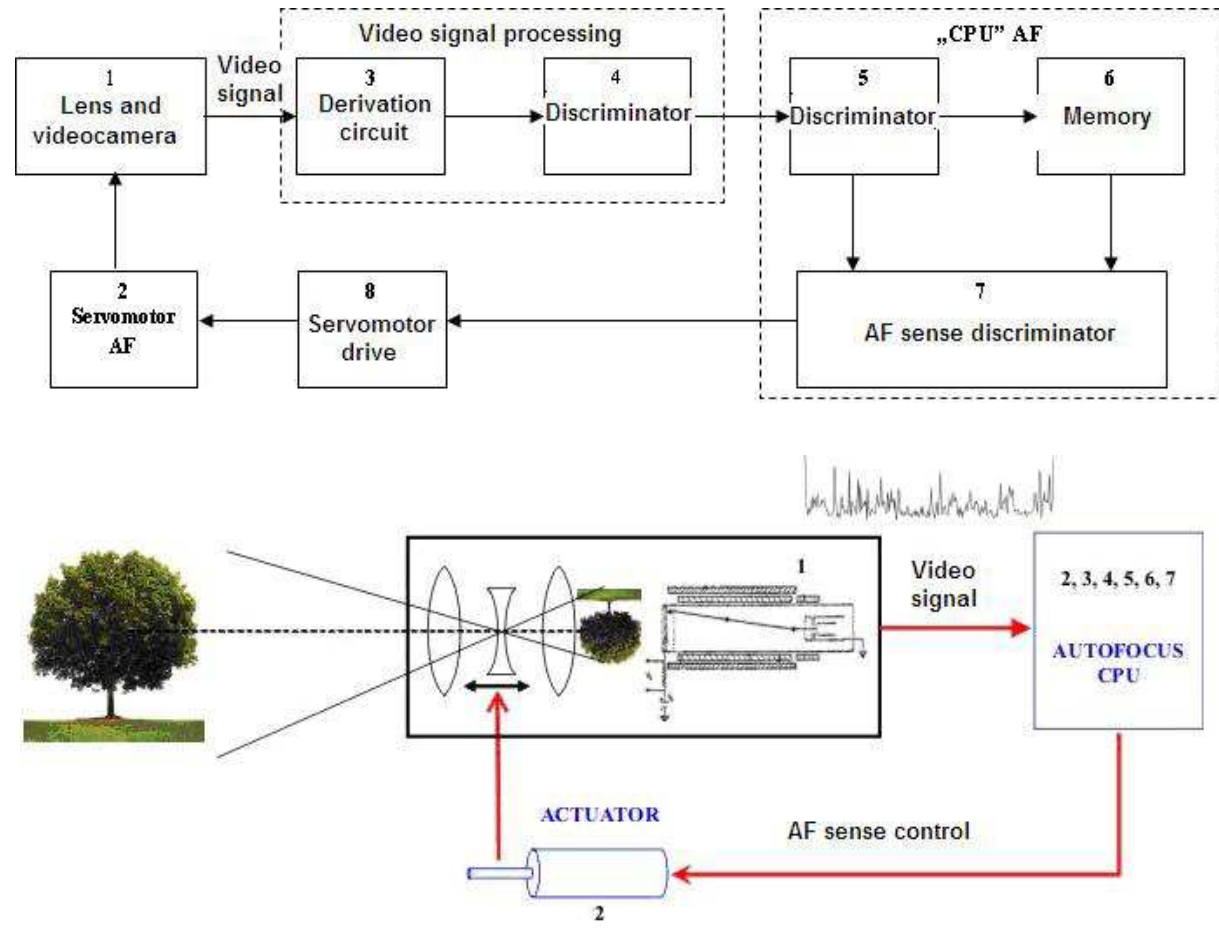

Figure 17. Block diagram of the invention, patent RO 44,277 /1966

"The convergence of the video camera objective 1 is modified in a certain direction by a servomotor 2 , which also implies the modification of the sharpness of the image formed in the camera; this image is explored and the obtained video signals are derived in a derivation circuit 3 . The obtained derivatives pass through two level discriminators 4 and 5 that memorize the derivative negative and infinite values (of very high value, exceeding a prescribed level), the signals obtained at the output of the discriminator are stored in a memory 6; these signals belong to an image of a certain sharpness.

After this first exploration of the image and video signal processing, another similar operation is performed, this time on an image of another sharpness, obtained by shifting the objective; the video signals corresponding to the same zone of the image obtained at the output of the discriminator 5 pass together with the corresponding signals from the memory 6 in another discriminator, where the derivative amplitudes are compared; the discriminator allows to pass only the derivatives with amplitudes higher than their correspondent (the derivatives will belong to either the first or the second image). The circuit 8 establishes the sense of modification of objective convergence or position with respect to the image plane that is situated on the front face of the video camera."

Figure 18 presents the diagram corresponding to two raster images; the first image is out-of focus, and the second image is correctly focused. In the diagram one can see the shape of the 
corresponding video signal, together with its derivatives.
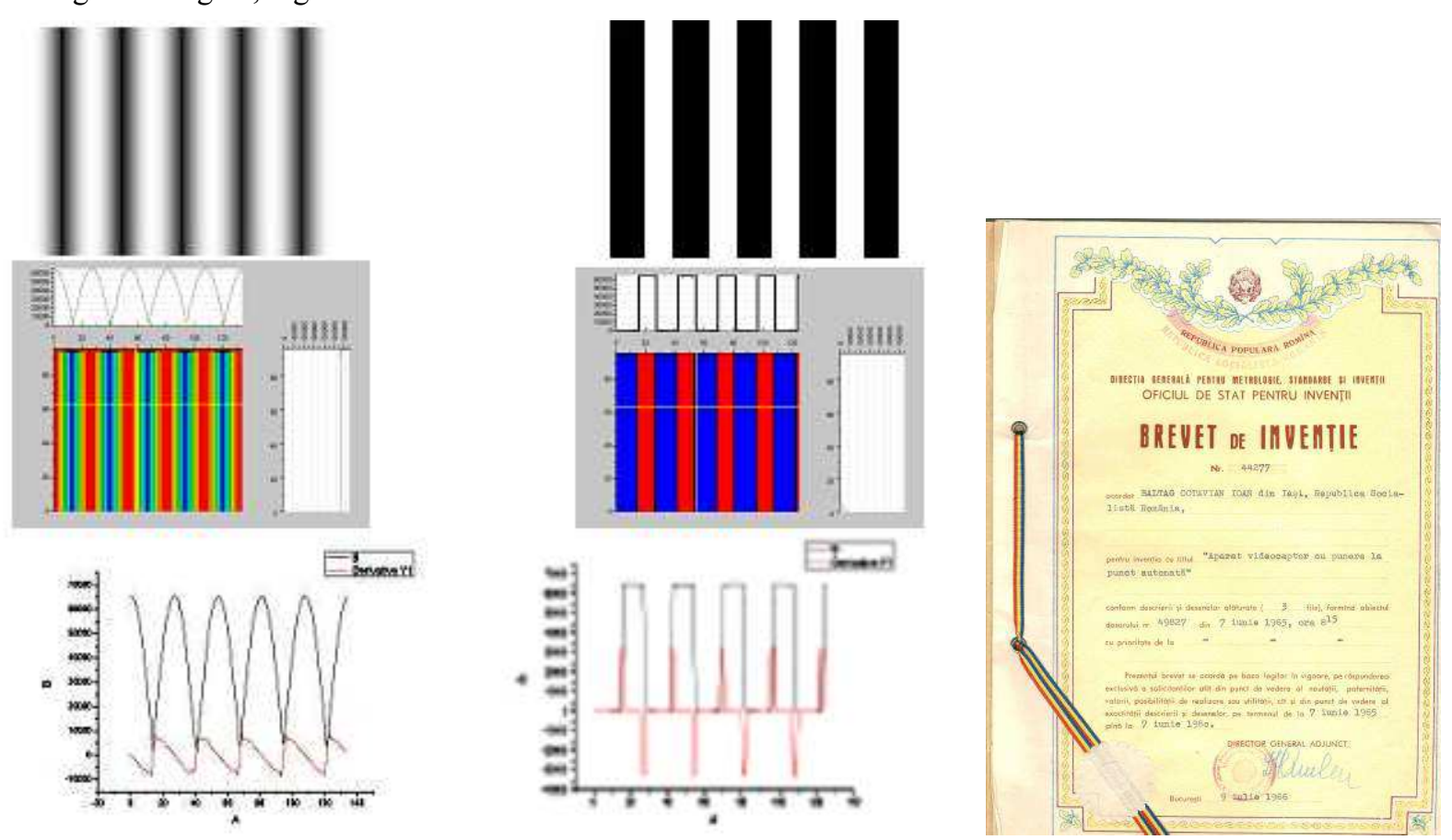

Figure 18. Diagram of the video signals and the patent

The analyzed video signals are used to control step by step the servomotor such that after the first command and reanalyzing the video signal the shifting sense is established. This shift is performed gradually until reaching the maximum contrast of the video signal, which corresponds to maximum optical contrast. The system operates like a system of extreme automation and adjustment. The advantage of the video signal analysis is that one can select for focusing the segment of the image which is of interest for the operator and the TV transmission. The analysis of the image contrast was used in the photographic and shooting cameras only after the years 80 's, when the integrated image sensors of the digital cameras appeared.

One can notice the difference between the amplitudes of the derivatives of the two video signals [48].

This patent makes the first mention of the image contrast analysis by means of the corresponding video signal [49].

\section{Conclusions}

In the first stage, the evolution of the AF systems was accomplished starting from the telemetry and optical triangulation systems (in different versions), especially using photoelectric sensors and signal analogue interpretation. The telemetry with ultra-acoustic sonar was applied by Polaroid, but it was not generalized.

A second important stage consisted in the utilization of the TCL technology by Honeywell, which permitted the utilization of SLR cameras.

The third stage in the AF development was marked by the analysis of the image contrast (contrast detection) and dedicated integrated photoelectric sensors.
A fourth important stage in AF was the utilization of image phase detection, using an auxiliary optic system and dedicated photoelectric sensors.

As for the analysis of the image contrast, a first mention of this method appears in the patent application "Videocaptor devices with automatic focusing" filed at OSIM (State Office for Inventions and Trademarks) Bucharest on the $7^{\text {th }}$ of June $1965,8^{\mathrm{h}} 15^{\mathrm{m}}$; the patent was granted on the $9^{\text {th }}$ of July 1966 , with the number 44,277 . The patent describes a solution which concerns a new method to obtain the image focusing in a camera tube using the dynamic analysis of the image contrast in a zone of the taken image selected by the operator.

\section{References}

[1] L. G. Simjian, "Self focusing camera" US Patent 1865 581, 1932.

[2] G. L. Beers, "Television pickup control system" US Patent 2 $403628,1946$.

[3] R. K. Orthuber, "Automatic focus adjuster" US Patent 2831 $057,1958$.

[4] H. W. Salinger, "Focusing adjusting system" US Patent $2838600,1958$.

[5] W. L. Steiner, "Automatic focus control system and method( ("US Patent 3211 831, 1965.

[6] O. Baltag, "Videocaptor devices with automatic focusing" Patent RO 44 177, 1966.

[7] F. Bidermaer, G. Fauth , F. Jakob, H. P. Simson, E. V. Wasilewski, "Camera focusing devices" US Patent 3274 914, 1966. 
[8] A. B. Pagel, “Automatic focusing system" US Patent 3442 193, 1969.

[9] C.E. Thomas, "Automatic focusing apparatus" US Patent 3450 $883,1969$.

[10] Martin, G.L., (1973). US Patent 3,708,619, Automatic focusing of optical system

[11] N. L. Stauffer, "Focus detecting apparatus" US Patent 4002 899, 1977.

[12] H. R. Everett, D.E. DeMuth, E. H. Stitz, "Survey of Collision Avoidance and Ranging Sensors for Mobile Robots", Technical Report 1194, Revision 1, Naval Command, Control and Ocean Surveillance Center RDT\&E Division, San Diego, California pp. 41-42, 1992.

[13] N.L. Stauffer, "Detecting apparatus for determining when image is in focus", US Patent 3836 772, 1974.

[14] N. L. Stauffer, "Detecting apparatus for determining when image is in focus", US Patent $3838275,1974$.

[15] N. L. Stauffer, "Auto focus camera", US Patent 3860 935, 1975.

[16] N. L. Stauffer, "Focus detecting apparatus", US Patent 3875 401, 1975.

[17] N. L. Stauffer, "Distance determining and automatic focusing apparatus”, US Patent 3958 117, 1976.

[18] N. L. Stauffer, “Autofocus system”, US Patent 4184 968, 1980.

[19] N. L. Staufer, "Range determination system", US Patent 4185 $191,1980$.

[20] N. L. Staufer, "Spatial low pass filter for range finding systems”, US Patent 4384 210, 1983.

[21] N. L. Staufer, „Digital autofocus system utilizing a photodetector array", US Patent 4317 991, 1982.

[22] D. J. Wilwerding, "Continuous focus proportional controller", US Patent 4207 461, 1980.

[23] D. J. Wilwerding, "Contrast sensing apparatus for automatic focus systems", US Patent 4247 762, 1981.

[24] D. J. Wilwerding, "Slit lens autofocus system", US Patent 4 $812640,1989$.

[25] G. O. Reynolds, "Focal sensor having optical low pass filter", US Patent 4474 446, 1984.

[26] I. R. Abel, "Autofocus system for infrared imaging device”, US Patent 4593 322, 1986.

[27] M. Cavina, "Nikon AF-Nikkor $80 \mathrm{~mm}$ f/4,5 del 1971. Rivelazioni, dati, schemi ottici e meccanici, inediti relativi ad una pietra miliare della tecnica fotografica", 2009, (accessed December 2014$\}$, retrieved from: http://www.nital.it/

[28] U. A. Tumen, "Faz algilamali otomatik netliğin tarihçesi", 2012 , http://uatumen.blogspot.ro/2012/03/otomatik-netligin-af-tarihc esi.html
[29] N. Goldberg, Camera Technology, Academic Press, Inc., San Diego pp. 38-39, 1992,

[30] S. Halgand, S., (accessed December 2014), retrieved from: http://www.collection-appareils.fr/x/html/page_standard.php?i d_appareil=1519

[31] C. H. Biber, "Rangefinding and focusing system for photographic cameras and the like" US Patent 3 522 764, 1970.

[32] R. Cassinis, P. Venuti, "Sonar Range Data Processing and Enhancement", Universita di Udine, Italy, Internal Report 87/036, (accessed in December 2014), retrieved from: http://www.ing.unibs.it/ arl/docs/papers/03_012.pdf

[33] J. Borenstein, H. R. Everett, L. Feng, "Where am I? Sensors and Methods for Mobile Robot Positioning", Univ. of Michigan, 1996.

[34] E. K. Shenk, “Auto focus movie camera”, US Patent 4294 532, 1981.

[35] S. Tamara, et all, "Autofocus camera", US Patent 4400071 , 1983.

[36] K. Araki, "Automatic focusing position detection apparatus", US Patent 4387 975, 1983.

[37] M. Eguki, “Automatic focusing devices”, US Patent 4381 523, 1983.

[38] M. Eguchi, et al., "Automatic focusing devices", US Patent 4 $423935,1984$.

[39] M. Matsuda, et al., "Camera autofocusing devices", US Patent 4199235,1980

[40] Ibid. [12] pp. 35-36.

[41] Y. Tomori, Y., "Automatic focus camera with interchangeable lens", US Patent 4400 075, 1983.

[42] Y. Nanba, "Focus detecting device", US Patent 3938 894, 1976.

[43] Y. Nanba, "Focus detecting photoelectric device" US Patent 4 $039824,1977$.

[44] T. Imura, et al, "Automatic focusing system for use in camera", US Patent 4182 555, 1980.

[45] M. Matsuda, et al, , "Rangefinding system", US Patent 4251 $144,1981$.

[46] S. Tamura, et al, "Distance measuring device", US Patent 4274 $735,1981$.

[47] O. Baltag, "Focalizarea Automata - Istoric (A Hystory of Autofocus", The 16-th International Conference Inventica 2012, Iasi, Romania, pp. 107-116, 2012.

[48] O. Baltag, "Focalizarea Automata - Istoric (The Autmatic Focusing - Hystory)", Romanian Journal of Industrial Property, no. 3-4, pp. 49-65, 2014.

[49] O. Baltag, Automatic Focusing - a Romanian Invention, Proc of the $41^{\text {st }}$ ICOHTEC Symposium "Technology in Times of Transition”, pp. 182, Brasov, Romania, 2014 